\title{
DETERMINAÇÃO DOS PARÂMETROS FISIOLÓGICOS E GRADIENTE TÉRMICO DE DIFERENTES GRUPOS GENÉTICOS DE CAPRINOS NO SEMI-ÁRIDO ${ }^{1}$
}

\author{
Determination of the physiologic parameters and thermal gradient \\ of different genetic groups of goats from semi-arid region
}

\author{
Expedito Danusio de Souza², Bonifácio Benício de Souza ${ }^{3}$, Wandrick Haus de Souza ${ }^{5}$, \\ Marcílio Fontes Cezar ${ }^{4}$, José Romulo Soares dos Santos ${ }^{6}$, Gustavo de Paula Tavares ${ }^{6}$
}

\section{RESUMO}

Objetivou-se com este trabalho avaliar o comportamento fisiológico de diferentes grupos genéticos de caprinos mediante respostas fisiológicas da temperatura retal, freqüência cardíaca e freqüência respiratória, e do gradiente entre temperatura retal e temperatura superficial e do gradiente entre temperatura superficial e temperatura ambiente, sob às condições do semi-árido. Foram utilizados trinta caprinos inteiros, sendo seis animais de cada grupo genético: $1 / 2$ Boer $+1 / 2$ SRD, $1 / 2$ Anglo-Nubiana $+1 / 2$ SRD, $1 / 2$ Savana + 1/2 SRD, 1/2 Kalarari + 1/2 SRD e 1/2 Moxotó + 1/2 SRD, com peso médio de 25,17 kg, distribuídos num delineamento inteiramente casualizado, em parcelas subdivididas no tempo. A alimentação consistiu numa ração composta de concentrado e volumoso. As variáveis ambientais registradas foram: temperatura máxima e mínima; temperatura do bulbo seco e bulbo úmido; temperatura do globo negro na sombra; umidade relativa do ar e ITGU. As tomadas dos parâmetros fisiológicos e das variáveis ambientais foram feitas às 9 horas e às 15 horas. No turno da tarde a freqüência respiratória foi significativamente $(P<0,05)$ mais elevada do que a da manhã. Não houve feito significativo $(P>0,05)$ de grupo genético e nem de turno para os parâmetros fisiológicos temperatura retal e freqüência cardíaca. Houve efeito significativo $(\mathrm{P}<0,05)$ para o gradiente térmico entre temperatura retal e superficial e temperatura superficial e temperatura do ambiente. Houve efeito significativo $(\mathrm{P}<0,05)$ para variação entre turno, para o gradiente térmico temperatura retal e superficial e para temperatura superficial e temperatura do ambiente. Os caprinos dos diferentes grupos genéticos apresentaram capacidade para manter a homeotermia.

Termos para indexação: temperatura superficial, temperatura retal, frequiência respiratória, freqüência cardíaca, caprinos.

\section{ABSTRACT}

This work was to evaluate the adaptation of different genetic groups of goats through the physiologic , the rectal temperature, heart frequency and breathing frequency of the thermal gradient parameters under the conditions of the semi-arid region. Thirty non-castred goats were used, being six animals of each genetic group: Boer $1 / 2+1 / 2$ WDR (without defined race), $1 / 2$ Anglo-Nubiana $+1 / 2$ WDR, $1 / 2$ Savana $+1 / 2$ WDR, $1 / 2$ Kalarari $+1 / 2$ WDR and $1 / 2$ Moxotó $+1 / 2$ WDR, with medium weight of $25.17 \mathrm{~kg}$, were assigned in a randomized in a split plot design in time. The feeding was done by a confinement ration the bulky. The meteorological maximum and minimum temperature, temperature of the dry bulb and humid bulb, temperature of the black globe in the shadow and relative humidity of the air were registred. The sockets of the physiologic parameters and of the environmental variables were done at 9:00h and the 15:00h. In the shift of the afternoon the breathing frequency was significantly $(\mathrm{P}<0.05)$ higher than the one of the shift of the morning, probably in function of the largest room temperature observed in this schedule. The data shown any significant $(\mathrm{P}>0.05)$ effect of genetic group also any effect of shift on the physiologic parameters such as rectal temperature and heart frequency. There was significant effect $(\mathrm{P}<0.05)$ for the gradient thermal rectal , superficial temperature and temperature of the atmosphere. There was significant effect $(\mathrm{P}<0,05)$, for variation among shift for the gradient on thermal rectal, superficial temperature and temperature of the atmosphere. The non-castred goatss from different genetic groups shown capacity to maintain the homeotermy.

Index terms: skin temperature, rectal temperature, breathing frequency, heart frequency, goats.

(Recebido para publicação em $1^{\circ}$ de outubro de 2003 e aprovado em 6 de janeiro de 2005)

\section{INTRODUÇÃO}

A produtividade ou mesmo a sobrevivência animal, depende principalmente de sua capacidade em manter a temperatura corporal dentro de certos limites.
Este processo denomina-se homeotermia, ou seja, a manutenção da temperatura corporal em níveis constantes, independentemente de variações da temperatura ambiente (JOHNSON, 1987).

\footnotetext{
1. Parte da Dissertação do primeiro autor a ser apresentada a UFPB/DZO - financiada pelo CNPq/Capes.

2. Mestrando em Zootecnia - UFPB/DZO, Professor da Escola Agrotécnica Federal de Iguatu, CE - 63.500-000 - Caixa Postal 38 - bolsista da CAPES, danusiosouza@yahoo.com.br

3. Professor Orientador - UFCG/DMV - Campus de Pados - bonif@cstr.ufcg.edu.br

4. Professor da UFCG/DMV - Patos, PB.

5. Pesquisador da EMEPA - João Pessoa, PB.

6. Estudante de Medicina Veterinária da UFCG - bolsista iniciação científica-PIBIC-CNPq/UFGC.
} 
Desta forma, a orientação sobre os sistemas de produção de caprinos deve considerar as variações climáticas desta região, já que o Nordeste esta situado na faixa tropical do planeta, definida como uma região semi-árida quente, onde a maior parte do seu território encontra-se compreendido dentro do polígono das secas (CARTER, 1976; MASON, 1980), predominando ainda as altas temperaturas do ar, conseqüência da elevada radiação solar incidente.

O impacto do calor sobre as variáveis fisiológicas resulta em um aumento percentual de 3,3 na temperatura retal e 194 na freqüência respiratória, com alterações, respectivamente, de $38,6^{\circ}$ para $39,9^{\circ} \mathrm{C}$ e de 32 para $94 \mathrm{mov} / \mathrm{min}$ (MCDOWELL, 1972). A freqüência respiratória alta pode ser uma maneira eficiente de perder calor por curtos períodos, mas caso mantida por várias horas, poderá resultar em sérios problemas para os animais. A respiração acelerada e contínua pode interferir na ingestão de alimentos e ruminação, adicionar calor endógeno a partir da atividade muscular e desviar a energia que poderia estar sendo utilizada em outros processos metabólicos e produtivos.

A temperatura corporal é o resultado do equilíbrio entre energia térmica produzida e energia térmica dissipada. (LEGATES et al., 1991), e a temperatura retal (TR) é a maneira mais fácil de estimá-la. Um aumento na TR significa que o animal está estocando calor, e se este não é dissipado, o estresse calórico manifesta-se.

A freqüência respiratória (FR) é também comumente usada como parâmetro para medir o estresse calórico. Assim, se uma FR alta for observada e o animal foi eficiente em eliminar o calor, poderá não ocorrer o estresse calórico (BERBIGIER, 1989).

Um trabalho realizado na UNESP, Campus de Botucatu - SP, utilizando cabras mestiças, secas adultas, Saanen x Nativa, submetidas a estresse térmico de 14 dias em câmara climática à temperatura de $36,8^{\circ} \mathrm{C}$ e $63 \%$ de umidade relativa, com radiação solar simulada das 10 horas às 14 horas, indicou um aumento significativo na freqüência respiratória de 27 para $39 \mathrm{mov} / \mathrm{min}$, para às 9 horas e 15 horas, respectivamente, em condições termoneutras e de 59 para $186 \mathrm{mov} / \mathrm{min}$ para às 9 horas e 15 horas, respectivamente, em condições de estresse térmico (GAYÃO et al., 1991).

Brasil (1997), trabalhando com cabras Parda Alpinas em lactação submetidas a estresse térmico, verificou médias de TR significativamente $(\mathrm{P}<0,05)$ mais elevadas à tarde $\left(39,97^{\circ} \mathrm{C}\right)$ do que pela manhã $\left(39,10^{\circ} \mathrm{C}\right)$. Lima (1983), trabalhando com caprinos da raça Moxotó, no semi-árido paraibano, também encon- trou efeito significativo $(\mathrm{P}<0,05)$ para turno, na qual os animais apresentaram um valor máximo de $38,8^{\circ} \mathrm{C}$ no período da manhã e de $39,30^{\circ} \mathrm{C}$ no período da tarde, para os caprinos do sexo feminino, enquanto que para os do sexo masculino, obteve $38,10^{\circ} \mathrm{C}$ pela manhã e $39,50^{\circ} \mathrm{C}$ à tarde Arruda e Pant (1984), trabalhando no Nordeste do Brasil, encontraram uma elevação de 1,48 e $2,03^{\circ} \mathrm{C}$ na temperatura retal, durante a tarde, em caprinos e ovinos, respectivamente. Anderson (1988) comenta que em animais que são normalmente ativos durante o dia, há uma variação normal da temperatura corporal que é mínima pela manhã, e máxima, no período da tarde. No entanto, sob estresse, notadamente no período da tarde, esta variação é muito marcante, evidenciando neste período uma hipertermia, fato este confirmado por Arruda e Pant $(1984,1985)$.

Arruda e Pant (1985), estudando a freqüência respiratória em caprinos pretos e brancos de diferentes idades, observaram que no período da tarde a freqüência respiratória foi maior e, que os caprinos de pelagem preta apresentaram maior frequiência respiratória que os brancos. Encontraram uma freqüência respiratória de 18,2 e 23,4 mov/min, manhã e tarde, respectivamente.

Reece (1988) afirma que a frequiência respiratória é um excelente indicador do estado de saúde, mas deve ser adequadamente interpretada, porque pode ser influenciada pela espécie, idade, exercícios, excitação e fatores ambientais.

A freqüência cardíaca é influenciada pela espécie, raça, idade, trabalho muscular e temperatura ambiente (KOLB, 1980).

A ingestão de grandes quantidades de alimento causa um aumento considerável na freqüência cardíaca, e a ruminação altera a freqüência cardíaca em $3 \%$. Nos caprinos normais, a freqüência cardíaca varia entre 70 a $90 \mathrm{bat} / \mathrm{min}$ (KELLY, 1976). Appleman e Delouche (1958) verificaram que caprinos vivendo em uma temperatura ambiente de $23^{\circ} \mathrm{C}$, apresentaram de 75 a 110 bat/min, com média de $89 \mathrm{bat} / \mathrm{min}$.

Os animais utilizam-se de mecanismos para manterem a homeotérmia, como a vasodilatação periférica, que aumenta o fluxo sangüíneo para a superfície corporal, aumentando a temperatura da superfície do animal (CHIMINEAU, 1983). Segundo Habeeb et al. (1992), o redirecionamento do fluxo sangüíneo e a vasodilatação facilitam a dissipação de calor por mecanismos não evaporativos (condução, convecção e radiação). Entretanto, a eficácia desses mecanismos depende do gradiente térmico entre o corpo do animal e o ambiente. Quando há um gradiente aceitável o excesso de calor 
corporal é dissipado do corpo aquecido para o meio mais frio, do contrário, o animal tem que utilizar mecanismos evaporativos como a sudorese e/ou freqüência respiratória (SOUZA et al., 2003).

\section{MATERIAL E MÉTODOS}

O experimento foi realizado na Estação Experimental de Pendência pertencente à Empresa Estadual de Pesquisa Agropecuária da Paraíba S/A (EMEPA), localizada no Município de Soledade-PB, na microrregião do Curimatau Ocidental, no Agreste Paraibano.

A média da temperatura máxima anual é de $35^{\circ} \mathrm{C}$ e a mínima é de $22^{\circ} \mathrm{C}$, com pequenas variações. A umidade relativa do ar situa-se em torno de 50\%. A precipitação pluviométrica apresenta uma amplitude de variação de 104 à 705mm/ano.

Os animais do experimento foram obtidos pelo programa de melhoramento para caprinos de corte, desenvolvido pela EMEPA, com o apoio do Banco do Nordeste e do CNPq, utilizando os recursos genéticos disponíveis na região, mediante estratégias de cruzamento.

Foram utilizados 30 animais inteiros, com peso médio de $25,17 \mathrm{~kg}$, sendo seis animais de cada grupo genético ( $1 / 2$ Boer + 1/2 SRD), (1/2 Anglo-Nubiana + 1/2 SRD), (1/2 Savana + $1 / 2$ SRD), ( $1 / 2$ Kalarari + 1/2 SRD) e ( $1 / 2$ Moxotó + $1 / 2$ SRD).

A dieta foi composta por feno de maniçoba (Manihot glaziowii), forrageira nativa da caatinga, mais uma mistura concentrada (milho, soja, óleo vegetal e sal mineral). A ração concentrada foi formulada visando obter-se uma dieta com alto valor nutritivo $73,1 \%$ de NDT e $18,6 \%$ de PB. Os dados da dieta concentrada e do feno em valores percentuais, encontram-se na Tabela 1.

Durante a fase experimental foram coletados as seguintes variáveis ambientais: temperatura máxima ( $\left.\mathrm{T}_{\text {máx }}\right)$ e temperatura mínima $\left(\mathrm{T}_{\text {mín }}\right)$; temperatura do bulbo seco (Tbs) e temperatura do bulbo úmido (Tbu); umidade relativa (UR); temperatura do globo negro (Tgn) e o índice de temperatura e umidade (ITGU). Essas variáveis foram coletadas nos mesmos dias e horários das variáveis fisiológicas.

Os cinco tratamentos foram distribuídos no delineamento inteiramente casualizado, com seis repetições, em parcelas subdivididas no tempo (STELL e TORRIE, 1980). As parcelas foram constituídas pelas cinco raças e as subparcelas pelos turnos. As médias foram comparadas pelo teste de Tukey a $1 \%$ e a 5\% de probabilidade.

As dietas foram fornecidas de acordo com o consumo médio de $750 \mathrm{~g} / \mathrm{cab} / \mathrm{dia}$, sendo distribuídas às 9 horas e às 14 horas.

Os parâmetros avaliados foram: temperatura retal (TR), freqüência respiratória (FR) e freqüência cardíaca (FC).

A metodologia aplicada para obtenção da temperatura retal (TR) consistiu na introdução de um termômetro clínico veterinário, com escala até $44^{\circ} \mathrm{C}$, diretamente no reto do animal, a uma profundidade de $5 \mathrm{~cm}$, de forma que o bulbo ficasse em contato com a mucosa do animal, permanecendo por um período de dois minutos e o resultado da leitura expresso em graus centígrados $\left({ }^{\circ} \mathrm{C}\right)$ (BACCARI JÚNIOR, 1990).

TABELA 1 - Composição da dieta concentrada e do feno em termos de participação percentual dos ingredientes.

\begin{tabular}{|c|c|}
\hline Ingrediente & Percentual \\
\hline \multicolumn{2}{|l|}{ Concentrado } \\
\hline Farelo de milho & $50 \%$ \\
\hline Farelo de soja & $23 \%$ \\
\hline Farelo de trigo & $5 \%$ \\
\hline Óleo vegetal & $1 \%$ \\
\hline Sal mineral & $1 \%$ \\
\hline \multicolumn{2}{|l|}{ Volumoso } \\
\hline Feno de maniçoba & $20 \%$ \\
\hline Total & $100 \%$ \\
\hline
\end{tabular}


A metodologia aplicada para obtenção da freqüência respiratória (FR) foi realizada por meio da auscultação indireta das bulhas, com o auxílio de um estetoscópio flexível, ao nível da região laringo-traqueal, contando-se o número de movimentos durante 15 segundos, e o valor obtido foi multiplicado por 4 para determinação da freqüência respiratória em movimentos por minuto (mov/min).

A metodologia aplicada para obtenção da freqüência cardíaca (FC) foi realizada com o auxílio de um estetoscópio flexível, colocado diretamente na região torácica esquerda à altura do arco aórtico, contando-se o número de movimentos durante 15 segundos, e o valor obtido foi multiplicado por 4 para determinação da freqüência cardíaca em batimentos por minuto (bat/min).

\section{RESULTADOS E DISCUSSÃO}

Os dados meteorológicos observados durante 0 período experimental encontram-se na Tabela 2. Os parâmetros fisiológicos observados: TR, FR e FC encontram-se na Tabela 3.

A temperatura retal (TR), apresentou efeito significativo $(\mathrm{P}<0,05)$ para turno conforme se observa na Tabela 3, fato também confirmado por Arruda e Pant (1985), os quais verificaram que a temperatura corporal dos caprinos no período da tarde foi significativamente $(\mathrm{P}<0,05)$ maior do que no período da manhã, em diferentes idades estudadas.

A média da temperatura retal dos animais de cada grupo foi semelhante a média geral encontrada por Silveira (1999), que foi de $39,37 \pm 0,99^{\circ} \mathrm{C}$, trabalhando com caprinos das raças Boer e Anglo-Nubiana no semiárido paraibano.
As médias da freqüência respiratória (FR) encontram-se na Tabela 3. Verifica-se que os animais do grupo genético $1 / 2$ Boer $+1 / 2$ SRD apresentaram uma freqüência respiratória significativamente $(\mathrm{P}<0,05)$ mais elevada do que os animais dos outros grupos genéticos. Fato também observado por Silveira (1999), que trabalhando com caprinos das raças Bôer e Anglo-Nubiana no semi-árido paraibano, verificou que os animais da raça Bôer demonstraram frequiência respiratória significativamente $(\mathrm{P}<0,05)$ mais elevada do que os da raça Anglo-Nubiana.

A diferença entre as freqüências respiratórias dos grupos genéticos pode estar relacionada com a individualidade de cada grupo, já que os animais dos grupos genéticos $1 \frac{1}{2}$ Anglo-Nubiana x SRD e $1 / 2$ Moxotó x SRD tiveram uma frequiência respiratória mais baixa. Os valores encontrados neste experimento para a FR foram mais elevados do que aqueles encontrados por Medeiros et al. (1989), quando foi obtido 26,90 mov/min para os caprinos da raça Anglo-Nubiana. E também mais elevado do que os obtidos por Silveira (1999), em que os caprinos da raça Boer apresentaram 38,83 \pm 12,02 $\mathrm{mov} / \mathrm{min}$ e os da raça Anglo-Nubiana apresentaram $54,19 \pm 12,40 \mathrm{mov} / \mathrm{min}$ nas condições do semi-árido paraibano.

Lima (1982), trabalhando com caprinos da raça Moxotó, concluiu-se que, a temperatura ambiente influi significativamente sobre a freqüência respiratória, nos períodos da manhã e tarde, efeito este que também foi constatado neste experimento.

O grupo genético $1 \frac{1}{2}$ Boer $+1 / 2$ SRD por ter sentido os efeitos da variação climática, foi o grupo que mais utilizou o mecanismo de aumento da FR, para manter a sua homeotermia nas condições experimentais, como consta na Tabela 3.

TABELA 2 - Média das variáveis ambientais coletadas durante o período experimental.

\begin{tabular}{l|c|c|c}
\hline \multirow{2}{*}{ Variáveis ambientais } & \multicolumn{2}{c|}{ TURNOS } & \multirow{2}{*}{ Média diária } \\
\cline { 2 - 3 } & Manhã & Tarde & \\
\hline Temperatura máxima, Tmáx $\left({ }^{\circ} \mathrm{C}\right)$ & - & - & 31,86 \\
Temperatura mínima, Tmín $\left({ }^{\circ} \mathrm{C}\right)$ & - & - & 20,00 \\
Temperatura do bulbo seco, Tbs $\left({ }^{\circ} \mathrm{C}\right)$ & 21,28 & 28,85 & 25,06 \\
Temperatura do bulbo úmido, Tbu $\left({ }^{\circ} \mathrm{C}\right)$ & 16,28 & 19,50 & 17,89 \\
Umidade relativa, UR $(\%)$ & 61,00 & 41,00 & 51,00 \\
Temperatura do globo negro, Tgn $\left({ }^{\circ} \mathrm{C}\right)$ & 24,71 & 32,43 & 28,57 \\
Índice de temperatura e umidade, ITGU & 71,25 & 79,15 & 75,20 \\
\hline
\end{tabular}

Ciênc. agrotec., Lavras, v. 29, n. 1, p. 177-184, jan./fev. 2005 
Determinação dos parâmetros fisiológicos e gradiente térmico de...

181

TABELA 3 - Médias \pm desvios-padrão das variáveis fisiológicas coletadas dos animais mestiços nativos e exóticos durante o período experimental.

\begin{tabular}{|c|c|c|c|}
\hline Grupo genético (GG) & $\operatorname{TR}\left({ }^{\circ} \mathrm{C}\right)$ & FR (mov/min) & FC (bat/min) \\
\hline 1/2 Bôer + 1/2 SRD & $39,58 \pm 0,19 \mathrm{a}$ & $58,67 \pm 14,22 \mathrm{a}$ & $126,32 \pm 10,04 \mathrm{a}$ \\
\hline $1 / 2$ Savana + 1/2 SRD & $39,61 \pm 0,23 \mathrm{a}$ & $52,61 \pm 10,86 \mathrm{ab}$ & $133,06 \pm 9,60 \mathrm{a}$ \\
\hline $1 / 2$ Kalarari + 1/2 SRD & $39,60 \pm 0,18 a$ & $54,47 \pm 11,83 \mathrm{ab}$ & $123,36 \pm 10,90 \mathrm{a}$ \\
\hline $1 / 2$ Anglo Nubiana + 1/2 SRD & $39,43 \pm 0,30 \mathrm{a}$ & $45,47 \pm 16,02 \mathrm{~b}$ & $124,75 \pm 11,39 \mathrm{a}$ \\
\hline 1/2 Moxotó + 1/2 SRD & $39,63 \pm 0,28 \mathrm{a}$ & $45,95 \pm 11,77 \mathrm{~b}$ & $121,36 \pm 10,80 \mathrm{a}$ \\
\hline \multicolumn{4}{|l|}{ Turnos } \\
\hline Manhã & $39,46 \pm 0,20 \mathrm{a}$ & $42,30 \pm 8,56 b$ & $127,96 \pm 10,34 \mathrm{a}$ \\
\hline Tarde & $39,68 \pm 0,23 \mathrm{a}$ & $60,57 \pm 11,48 \mathrm{a}$ & $125,18 \pm 11,36 \mathrm{a}$ \\
\hline $\mathrm{CV}$ & 0,47 & 15,43 & 4,31 \\
\hline
\end{tabular}

FR - Freqüência respiratória, FC - Freqüência cardíaca, TR - Temperatura retal

CV - Coeficiente de variação

Media seguidas de mesma letra não diferem significativamente pelo teste de Tukey a $5 \%$ de probabilidade.

As médias da freqüência cardíaca (FC) encontram-se na Tabela 3. Verifica-se que não houve diferença significativa $(\mathrm{P}>0,05)$ entre os diferentes grupos genéticos e entre os turnos estudados. Resultados contrários foram verificados por Kaushish et al. (1987), que observaram a influência da temperatura ambiente sobre a variável FC em caprinos.

Os valores da freqüência cardíaca, dos diferentes grupos genéticos, encontrados neste experimento estão acima daqueles encontrados por Azevedo (1982), que trabalhando com cabras da raça Moxotó em regime semiintensivo no semi-árido paraibano, obteve valores de freqüência cardíaca para cabras secas e não gestantes de $84,20 \pm 0,63 \mathrm{bat} / \mathrm{min}$ e por Silveira (1999), que trabalhando com caprinos da raça Boer e Anglo-Nubiana também em regime semi-intensivo no semi-árido paraibano, que obteve uma média de 84,83 \pm 16,46 bat/min para as duas raças.

Mesmo não havendo diferença significativa entre os grupos genéticos para a variável frequiência cardíaca (FC) neste experimento, Arruda e Pant $(1984,1985)$ e Medeiros et al. (1998) trabalhando com caprinos encontraram diferenças entre raças para a variável FC. Silveira (1999) verificou em trabalho realizado com caprinos das raças Boer e Anglo-Nubiana, que o efeito do turno para a variável freqüência cardíaca no período foi significativa $(\mathrm{P}<0,05)$, já que a FC foi mais elevada no perí- odo da tarde do que no período da manhã, possivelmente, atribuído às diferenças na temperatura ambiente.

Detweiler (1988) afirma que os dados de freqüências cardíacas na literatura científica, na maioria das vezes, são discrepantes, devido às diferentes condições ambientais em que foram obtidas. Destaca-se ainda como limites, uma freqüência cardíaca de 70 a 80 bat/min para caprinos e ovinos no repouso.

As médias do gradiente entre temperatura retal e temperatura superficial (TR-TS) e do gradiente entre temperatura superficial e temperatura do ambiente (TS -TA) estão na Tabela 5.

No turno da manhã o grupo genético $1 / 2$ Moxotó $+1 / 2$ SRD apresentou maior gradiente térmico entre as temperaturas retal e superficial $(\mathrm{P}<0,05)$ em relação aos grupos genéticos $1 / 2$ Savana + 1/2 SRD, $1 / 2$ Kalarari + $1 / 2$ SRD e $1 / 2$ Anglo Nubiana $+1 / 2$ SRD não diferindo do grupo genético $1 / 2$ Boer $+1 / 2 \mathrm{SRD}$.

$\mathrm{O}$ gradiente térmico entre as temperaturas retal e superficial, pela manhã, não revelou significância $(\mathrm{P}>0,05)$ entre os grupos genéticos $1 / 2$ Savana $+1 / 2$ SRD, $1 / 2$ Kalarari $+1 / 2$ SRD e $1 / 2$ Anglo-Nubiana + $1 / 2$ SRD, entretanto, o grupo genético $1 / 2$ Boer $+1 / 2$ SRD apresentou significância $(\mathrm{P}<0,05)$ em relação a esses grupos, não diferindo do grupo genético $1 / 2$ Moxotó + 1/2 SRD e $1 / 2$ Anglo Nubiana + $1 / 2 \mathrm{SRD}$.

Ciênc. agrotec., Lavras, v. 29, n. 1, p. 177-184, jan./fev. 2005 
TABELA 5 - Média dos gradientes térmicos (TR - TS ) e (TS - TA) em função dos grupos genéticos e dos turnos.

\begin{tabular}{lcccc}
\hline \multirow{2}{*}{ Grupo genético } & \multicolumn{2}{c}{ TR-TS } & \multicolumn{2}{c}{ TS-TA } \\
\cline { 2 - 5 } & Manhã & Tarde & Manhã & Tarde \\
\hline $1 / 2$ Bôer + 1/2 SRD & $11,89 \mathrm{bcA}$ & $8,66 \mathrm{aB}$ & $6,33 \mathrm{acA}$ & $2,15 \mathrm{aB}$ \\
$1 / 2$ Savana $+1 / 2$ SRD & $11,29 \mathrm{aA}$ & $8,29 \mathrm{abB}$ & $6,93 \mathrm{bA}$ & $2,58 \mathrm{abB}$ \\
$1 / 2$ Kalarari $1 / 2+$ SRD & $11,51 \mathrm{aA}$ & $8,42 \mathrm{abB}$ & $6,76 \mathrm{bcA}$ & $2,38 \mathrm{abB}$ \\
$1 / 2$ Anglo Nubiana $+1 / 2$ SRD & $10,99 \mathrm{abA}$ & $7,99 \mathrm{bB}$ & $7,02 \mathrm{bA}$ & $2,73 \mathrm{bB}$ \\
$1 / 2$ Moxotó $+1 / 2$ SRD & 12,24cA & $7,14 \mathrm{abB}$ & $5,93 \mathrm{aA}$ & $2,81 \mathrm{bB}$ \\
\hline \multicolumn{1}{c}{ CV } & \multicolumn{3}{c}{5,07} \\
\hline
\end{tabular}

TR = temperatura retal, $\mathrm{TS}=$ temperatura superficial e $\mathrm{TA}=$ Temperatura ambiental. $\mathrm{CV}=$ coeficiente de variação.

Médias seguidas de mesma letra maiúscula na linha, e minúscula na coluna não diferem significativamente pelo teste Tukey a $5 \%$ de probabilidade.

No turno da tarde o grupo genético $1 / 2$ Bôer + $1 / 2$ SRD apresentou maior gradiente térmico entre as temperaturas retal e superficial $(\mathrm{P}<0,05)$, em relação aos grupos genéticos $\quad 1 / 2$ Anglo-Nubiana $+1 / 2 \mathrm{SRD}$, não diferindo dos demais.

$\mathrm{O}$ gradiente entre a temperatura superficial e temperatura do ambiente, pela manhã, não revelou significância $(\mathrm{P}>0,05)$ entre os grupos genéticos $1 / 2$ Boer + $1 / 2$ SRD e $1 / 2$ Moxotó + 1/2 SRD, mas em relação aos demais grupos houve diferença significativa $(\mathrm{P}<0,05)$. Os animais dos grupos genéticos $1 / 2$ Savana $+1 / 2$ SRD, $1 / 2$ Kalarari $1 / 2$ + SRD e $1 / 2$ Anglo-Nubiana + 1/2 SRD também não diferiram estatisticamente $(\mathrm{P}>0,05)$ entre si, mas apresentaram significância $(\mathrm{P}<0,05)$ para os demais grupos. Os animais do grupo genético $1 / 2$ Bôer + $1 / 2$ SRD e $1 / 2$ Kalarari + 1/2 SRD não diferiram estatisticamente $(P>0,05)$ entre si, mas apresentaram significância $(\mathrm{P}<0,05)$ em relação aos outros grupos.

No turno da tarde os animais do grupo genético $1 / 2$ Boer $+1 / 2$ SRD apresentaram um menor gradiente $(\mathrm{P}<0,05)$ entre temperatura superficial e temperatura do ambiente, em relação aos animais dos grupos genéticos $1 / 2$ Anglo-Nubiana + 1/2 SRD e 1/2 Moxotó + 1/2 SRD, não diferindo estatisticamente $(\mathrm{P}>0,05)$ dos demais grupos. Os animais dos grupos genéticos $1 / 2$ Anglo-Nubiana + $1 / 2$ SRD e $1 / 2$ Moxotó + 1/2 SRD apresentaram um maior gradiente $(\mathrm{P}<0,05)$ entre temperatura superficial e temperatura do ambiente em relação ao grupo genético $1 / 2$ Boer $+1 / 2$ SRD, não diferindo estatisticamente $(P>0,05)$ dos demais grupos.
As médias do gradiente térmico entre temperatura superficial e temperatura do ambiente do turno da tarde revelaram significância $(\mathrm{P}<0,05)$ em relação ao turno da manhã.

Por ter apresentado um menor gradiente térmico entre a temperatura superficial e do ambiente, como pode ser observado na Tabela 5, o grupo genético $1 / 2$ Bôer + $1 / 2$ SRD teve que aumentar a freqüência respiratória, como observado na Tabela 2, para manter a homeotermia, portanto, sendo considerado menos adaptado, nas condições experimentais.

Os animais dos grupos genéticos $1 / 2$ AngloNubiana $+1 / 2$ SRD e $1 / 2$ Moxotó + $1 / 2$ SRD, foram considerados mais adaptados, nas condições experimentais, já que apresentaram um maior gradiente térmico entre a temperatura superficial e do ambiente, como observado na Tabela 5, e uma menor freqüência respiratória em relação aos demais grupos (Tabela 2).

\section{CONCLUSÕES}

Os resultados obtidos mediante as variáveis fisiológicas e ambientais, permitiram concluir que:

Os animais do grupo genético $1 / 2$ Boer $+1 / 2$ SRD para manter a sua homeotermia utilizaram-se de um aumento da freqüência respiratória superior aos mestiços $1 \frac{1}{2}$ Anglo-Nubiana + 1/2 SRD e 1/2 Moxotó + 1/2 SRD.

Os animais do grupo genético $1 / 2$ Anglo-Nubiana $+1 / 2$ SRD e do grupo genético $1 \frac{1}{2}$ Moxotó + $1 / 2$ SRD por manterem a sua homeotermia com uma freqüência res- 
piratória mais baixa podem ser classificados como mais adaptados às condições experimentais.

No estudo do gradiente térmico, os animais dos grupos genéticos $1 / 2$ Anglo-Nubiana $+1 / 2$ SRD e $1 / 2$ Moxotó + $1 / 2$ SRD mostraram-se mais adaptados e os animais do grupo genético $1 / 2$ Bôer + $1 / 2$ SRD menos adaptado, nas condições experimentais.

\section{REFERÊNCIAS BIBLIOGRÁFICAS}

ANDERSON, B. E. Regulação da temperatura e fisiologia ambiental. In: DUKES, H. H.; SWENSON, M. J. Fisiologia dos animais domésticos. 10. ed. Rio de Janeiro: Guanabara Koogan, 1988. p. 623-629.

APPLEMAN, R. D.; DELOUCHE, J. C. Behavioral, phisiological and responses of goats to temperature $0^{\circ}$ to $40^{\circ} \mathrm{C}$. Journal AnimalScience, Champaign, v. 17, n. 2, p. 326-335, 1958.

ARRUDA, F. A. V.; PANT, K. P. Tolerância ao calor de caprinos e ovinos sem lã em Sobral. Pesquisa Agropecuária Brasileira, Brasília, v. 19, n. 3, p. 379-385, 1984.

ARRUDA, F. A. V.; PANT, K. P. Freqüência respiratória em caprinos pretos e brancos de diferentes idades. Pesquisa Agropecuária Brasileira, Brasília, v. 20, n. 11, p. 1351-1354, 1985.

AZEVEDO, S. A. Estudos da freqüência cardíaca e da temperatura corporal de cabras (Capra hircus, L.), da raça Moxotó e suas modificações influenciadas pela gestação e lactação. 1982. 52 f. Dissertação (Mestrado) - Universidade Federal Rural de Pernambuco, Recife, 1982.

BACCARI JÚNIOR, F. Métodos e técnicas de avaliação da adaptabilidade dos animais às condições tropicais. In: SIMPÓSIO INTERNACIONAL DE BIOCLIMATOLOGIA ANIMAL NOS TRÓPICOS: PEQUENOS E GRANDES RUMINANTES, 1., 1990, Sobral-CE. Anais... Sobral: EMBRAPA-CNPC, 1990. p. 9-17.

BERBIGIER, P. Effect of heat on intensive meat production in the tropics: cattle, sheep and goats, pigs. In: CICLO INTERNACIONAL DE PALESTRAS SOBRE BIOCLIMATOLOGIA ANIMAL, 1., 1989, Botucatu. Anais... Jaboticabal: FMVZ/UNESP/FUNEP, 1989. p. 7-44.
BRASIL, L. H. A. Efeitos do estresse térmico sobre a produção, comparação química do leite e respostas termorreguladoras de cabras Pardas Alpinas. 1997. 78 f. Tese (Doutorado em Nutrição e Produção Animal) - Faculdade de Medicina Veterinária e Zootecnia, Universidade Estadual Paulista, Botucatu, 1997.

BRAZ, M. B. Seminologia médica animal. Lisboa: Fundação Calouste Gulbenkian, 1971. v. 2.

CARTER, E. D. Project report: a review of proposals for, strenghtening agricultural and livestock reserch with special reference to a goat and sheep project for North - East Brazil. Osmond: University Adelaide Gled Osmond, 1976. 20 p.

CHIMINEAU, P. Médio ambiente y reproducción animal. World Animal Review, Roma, v. 77, n. 1, p. 2-14, 1993.

DETWEILER, D. R. Regulação cardíaca. In: DUKES, H. H.; SWENDSON, M. J. Fisiologia dos animais domésticos. 10. ed. Rio de Janeiro: Guanabara Koogan, 1988. p. 133-143.

GAYÃO, A. L. B. A.; BACCARI JÚNIOR, F.; MASSONE, F. Respostas termorreguladoras de cabras mestiças Saanen-nativas submetidas ao stress térmico de curta duração. In: REUNIÃO ANUAL DA SOCIEDADE BRASILERA DE ZOOTECNIA, 28., 1991, João Pessoa-PB. Anais... João Pessoa: SBZ, 1991. p. 492.

HABEEB, A. L. M.; MARAY, I. F. M.; KAMAL, T. H. Farm animals and the environment. Cambridge: CAB, 1992. 428 p.

JOHNSON, H. D. Bioclimatology and adaptation of livestock. Amsterdam: Elsevier, 1987. 279 p.

KAUSHISH, S. K.; GEORGE, G. C.; SENGUPTA, B. $P$. Effect of heat and water restriction on physiological responses of Beetal and Black Bengal goats. Indian Journal Animal Science, New Delhi, v. 57, n. 5, p. 461-465, 1987.

KELLY, W. R. Diagnóstico clínico veterinário. 2. ed. Barcelona: Continental, 1976. 444 p.

KOLB, E. Coração e circulação. In: Fisiologia veterinária. 4. ed. Rio de Janeiro: Guanabara Koogan, 1980. p. 293-294. 
LEGATES, J. E. et al. Body temperature and respiratory rate of lactating dairy cattle under field and chamber conditions. Journal Dairy Science, Champaign, v. 74, p. 2491-2500, 1991.

LIMA, E. R. de. Estudo da freqüência respiratória e do rúmen em caprinos (Capra hircus, L.) da raça Moxotó, criadas no Estado da Paraíba. 1982. 48 f. Dissertação (Mestrado) - Universidade Federal Rural de Pernambuco, Recife, 1982.

LIMA, M. S. Correlação da temperatura corporal e volume globular de caprinos (Capra hircus, L.) normais da raça Moxotó. 1983. 68 f. Dissertação (Mestrado) - Universidade Federal Rural de Pernambuco, Recife, 1983.

MASON, I. L. Sheep and goat production in the drought polygon of Northeast Brazil. World Animal Review, Rome, v. 34, p. 23-28, 1980.

MCDOWELL, R. G. Improvement of livestock production in warm cllimates. San Francisco: Freeman, 1972. $711 \mathrm{p}$.

MEDEIROS, L. F. D.; COUTINHO, L. de S.; SOUSA, J. C. D. de. Estimativa da tolerância de calor em caprinos e ovinos. Arquivo da Universidade Federal Rural do Rio de Janeiro, Itaguaí, v. 12, n. 1-2, p. 6572, 1989.
MEDEIROS, L. F. D. et al. Reações fisiológicas de caprinos de diferentes raças mantidos à sombra, ao sol e em ambiente parcialmente sombreado. In: REUNIÃO ANUAL DA SOCIEDADE BRASILEIRA DE ZOOTECNIA, 35., 1998, Botucatu. Anais... Botucatu: SBZ, 1998, v. 1.

REECE, W. O. Respiração nos mamíferos. In: DUKES, H. H.; SWENSON, M. J. Fisiologia dos animais domésticos. 10. ed. Rio de Janeiro: Guanabara Koogan, 1988. p. 199-205.

SILVEIRA, J. O. de A. Respostas adaptativas de caprinos das raças Boer e Anglo-Nubiana às condições do semi-árido Brasileiro. 1999. $37 \mathrm{f}$. Dissertação (Mestrado) - Universidade Federal da Paraíba, Areia, PA, 1999.

SOUZA, B. B. Avaliação da adaptabilidade de ovinos de diferentes genótipos às condições climáticas do semiárido através de respostas fisiológicas e gradientes térmicos. In: CONGRESSO PERNAMBUCANO DE MEDICINA VETRINÁRIA, 5.; SEMINÁRIO NORDESTINO DE CAPRINO-OVINOCULTURA, 6., 2003, Recife. Anais... Recife: [s.n.], 2003. p. 281-282.

STEEL, R. D. G.; TORRIE, J. H. Principles and procedures of statistics: biometria approach. 2. ed. New York: McGraw-Hill Book, 1980. 633 p. 